\title{
Characteristics of dissolved organic matter in a water purification plant and distribution pipes
}

\author{
Toshiro Yamada ${ }^{1, *}$, Maulana Yusup Rosadi $^{2}$, Hudori ${ }^{3}$, Yukitaka Suzuki ${ }^{3}$, Eri Ito $^{4}$, and \\ Fusheng $\mathrm{Li}^{5}$ \\ ${ }^{1}$ Faculty of Engineering, Gifu University, 501-1193 Gifu, Japan \\ ${ }^{2}$ Graduate School of Natural Science and Technology, Gifu University, 501-1193 Gifu, Japan \\ ${ }^{3}$ Graduate School of Engineering, Gifu University, 501-1193 Gifu, Japan \\ ${ }^{4}$ Aichi Public Enterprise Bureau, Aichi Prefecture, 470-0151 Aichi, Japan \\ ${ }^{5}$ River Basin Research Center, Gifu University, 501-1193 Gifu, Japan
}

\begin{abstract}
Dissolved organic matter (DOM) needs to be characterized to determine the ability of a water treatment unit to reduce organic matter and its relationship to the levels of chlorine required for disinfection. We investigated water quality parameters, including DOM, in a water purification plant and distribution pipes located in central Japan. The dissolved organic carbon (DOC) concentration decreased from $0.73 \mathrm{mg} / \mathrm{L}$ in raw water to $0.50 \mathrm{mg} / \mathrm{L}$ in purified water by conventional treatment processes. The DOC of purified water decreased slightly in the pipes downstream of the plant. The three-dimensional excitation-emission matrix fluorescence spectra showed some specific peaks for humic-like substances and protein-like substances in all water samples, and the compositions of DOM in purified water were close to those in raw water. However, there was a peak of protein-like substances in purified and returned water, which was not observed for raw water. The fluorescence intensities of humic-like substances in purified water decreased downstream of the plant, while the fluorescence intensities of protein-like substances increased in the pipes. The measured molecular weight distributions of DOM in water presented some specific peaks, and DOM with a smaller molecular weight was not easily removed by water treatment processes. There were positive relationships between decreases in the intensities of some peaks and residual chlorine decay.
\end{abstract}

\section{Introduction}

In Japan, most drinking water is treated by conventional treatment methods, such as coagulation, sedimentation, and filtration. The treatment system can produce high-quality drinking water because the pollution level of raw water is low. However, some drinking water treatment units use a closed system that reuses water from sludge treatment and backwash filters. This can affect the quality of raw water, especially the content of organic matter.

\footnotetext{
*Corresponding author: ymd@gifu-u.ac.jp
} 
Natural organic materials (NOM) are complex compounds that are present in natural water and originate from the decomposition of organic material from plants and from byproducts from algae, bacteria, and aquatic plants [1]. The presence of NOM in drinking water can cause various problems, such as color, odor, taste, transport of pesticides, transport of heavy metals, and, most importantly, disinfection by-products (DBPs) [2]. In water distribution systems, the presence of NOM can cause microorganisms to grow in pipes. Conventional and advanced treatment can reduce NOM in drinking water. Conventional water treatment processes, such as coagulation, sedimentation, and filtration, can reduce organic matter, especially that of large molecular weight [3]. Advanced treatment methods, such as activated carbon, are required to reduce NOM with a low molecular weight. Membrane filtration is an advanced treatment method that reduces all NOM components [4].

Characterizing NOM is an important step in elucidating its concentration and component. NOM can be characterized by measuring several key parameters, such as dissolved organic carbon (DOC), adsorption of UV light (UV254), specific UV-absorbance (SUVA), molecular weight (MW), and excitation-emission matrix (EEM) fluorescence spectra. DOC can indicate the concentration of organic materials, but does not show the behavior of NOM during water treatment [5]. In Japan, UV260 is normally measured instead of UV254. SUVA is obtained by dividing the UV260 by the DOC concentration, and this ratio can describe the hydrophilicity and hydrophobicity of NOM. The SUVA value is correlated with the trihalomethane formation potential [6]. The measurement of MW is vital for determining the distribution of DOM sizes in water because it is connected to the performance of the water treatment plant and DBPs formation [7]. EEM is an alternative technique that is most popular for characterizing complex DOM by its fluorescent components because it is a cheaper, non-invasive technique that can cluster DOM fractions by their chemical properties [8].

In Japan, chlorine is added to tap water to prevent recontamination by microorganisms following water purification. However, advanced management strategies for chlorination, such as reducing chlorine doses, are required to solve problems such as chlorine odor and by-products of the chlorination process. It is vital that microbially-safe, nontoxic, and palatable water is delivered to consumers. The residual chlorine in tap water can be reduced by ammonia, iron, manganese, and dissolved organic matter, which are found in treated water. High chlorine concentrations can result in high DOC concentrations in tap water [3], and chlorination might increase the level of assimilable organic carbon (AOC) by breaking down macromolecules of NOM into smaller compounds [9]. This can stimulate microorganism growth in piping systems because chlorinated DBPs are easily absorbed by bacteria [10].

Residual chlorine concentrations in purified water decreased to a greater extent during summer at some supply points in a water supply system located in central Japan. As the concentrations of ammonia, iron, and manganese were relatively low, the DOM from water, including backwash and filtrate water from a sludge treatment process, returned to a receiving well in this purification plant was a likely cause of this problem. However, another study found that recycled water from filter backwash contains lower levels of UV254 and DOC concentrations, but higher turbidity and aluminum concentrations than raw water [11]. The recycling process of water treatment improves the coagulation efficiency, including organic material removal [12]. Furthermore, the contribution of the distribution pipe wall to the reduction in residual chlorine was considered small owing to the large diameters of the pipes. This study focuses on the characterization of DOM in a water purification plant, distribution pipes, and sludge filtrate, and examines the relationships between DOM and residual chlorine decay. 


\section{Methods}

\subsection{Sample collection}

Twenty-two water quality surveys were conducted in a water purification plant located in central Japan, and thirteen at supply points in the water supply system from April 2014 to January 2016. The sampling sites are shown in Fig. 1. Raw, purified, returned, and filtrate water from the sludge treatment process were collected from the plant. Water samples were collected at supply points A, B, C, and D in the water supply system, with average arrival times from the plant of approximately $7.5,13,23$, and 24 hours, respectively.

Samples were filtered using cellulose acetate membrane filters with pore sizes of 0.2 micrometers. Filtered samples were kept in a fridge at $5{ }^{\circ} \mathrm{C}$ before the DOC, UV260, dissolved organic nitrogen (DON), EEM, and MW distributions were analyzed.

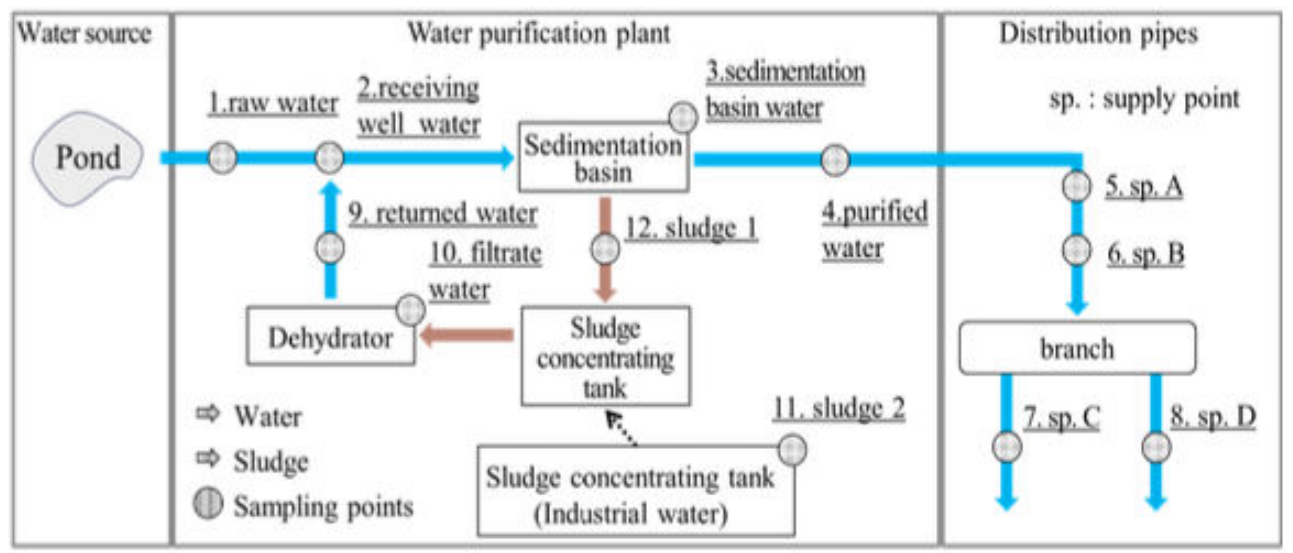

Fig. 1. Locations of sampling points.

\subsection{Analytical methods}

DOC and UV260 were analyzed using a TOC analyzer (TOC-Vws, Shimadzu, Japan) and a spectrophotometer (UV-1600 GLP, Shimadzu, Japan), respectively. Dissolved organic nitrogen (DON) was obtained as the difference between the total dissolved and total inorganic nitrogen, including ammonium, nitrite, and nitrate nitrogen, which were analyzed by ion chromatography.

The fluorescence EEM of samples was analyzed using a spectrofluorometer (RF-5300, Shimadzu, Japan). The excitation and emission scans observed wavelengths between 220 and $550 \mathrm{~nm}$ with $5 \mathrm{~nm}$ increments. The obtained fluorescence intensities of the samples were normalized using the quinine sulfate unit (QSU) by dividing the fluorescence intensity values of all samples by that of 10-ppb quinine sulfate (in a $0.05-\mathrm{M} \mathrm{H}_{2} \mathrm{SO}_{4}$ solution) at the designated excitation wavelength (Ex) of $350 \mathrm{~nm}$ and emission wavelength (Em) of 450 nm.

The molecular weight characteristics of DOM were evaluated at a wavelength of 260 $\mathrm{nm}$ using a high-performance size exclusion chromatography system that consisted of a silica chromatographic column (GL-W250-X, $10.7 \times 450 \mathrm{~nm}$, Hitachi) and a UV detector (Model LC-10AV, Shimadzu). Pure water containing $0.02 \mathrm{M}$ of $\mathrm{Na}_{2} \mathrm{HPO}_{4}$ and $0.02 \mathrm{M}$ of $\mathrm{KH}_{2} \mathrm{PO}_{4}$ was used as the eluent and was introduced to the column at a constant flow rate of $0.5 \mathrm{~mL} / \mathrm{min}$. 


\section{Results and Discussion}

\subsection{Characteristics of DOM in the water purification plant}

The average DOC concentrations in the raw and purified water were 0.73 and $0.50 \mathrm{mg} / \mathrm{L}$, respectively (Fig. 2). The DOM in the raw water decreased by approximately $30 \%$ through undergoing the water treatment processes in the plant. This reduction is higher than that achieved by some conventional water treatment systems in Taiwan and Spain, which achieved a decrease of $14-18 \%[2,4]$, but lower than that of conventional water treatment systems in Australia and Norway, which achieved reductions of approximately 59\% [16]. As the SUVA (UV260/DOC) of raw water decreased by approximately $60 \%$, it is likely that humic-like substances were preferentially removed by the water treatment processes in the plant. Humic-like substances and compounds with a high MW are more easily removed by conventional treatment through bridging or sweep flocculation [1]. The DOC concentration in the returned water was approximately $30 \%$ higher than that of the raw water, while the SUVA of the returned water was approximately $75 \%$ lower than that of raw water. The low level of SUVA indicates that returned water mostly contains non-humic substance and low-MW compounds [1].

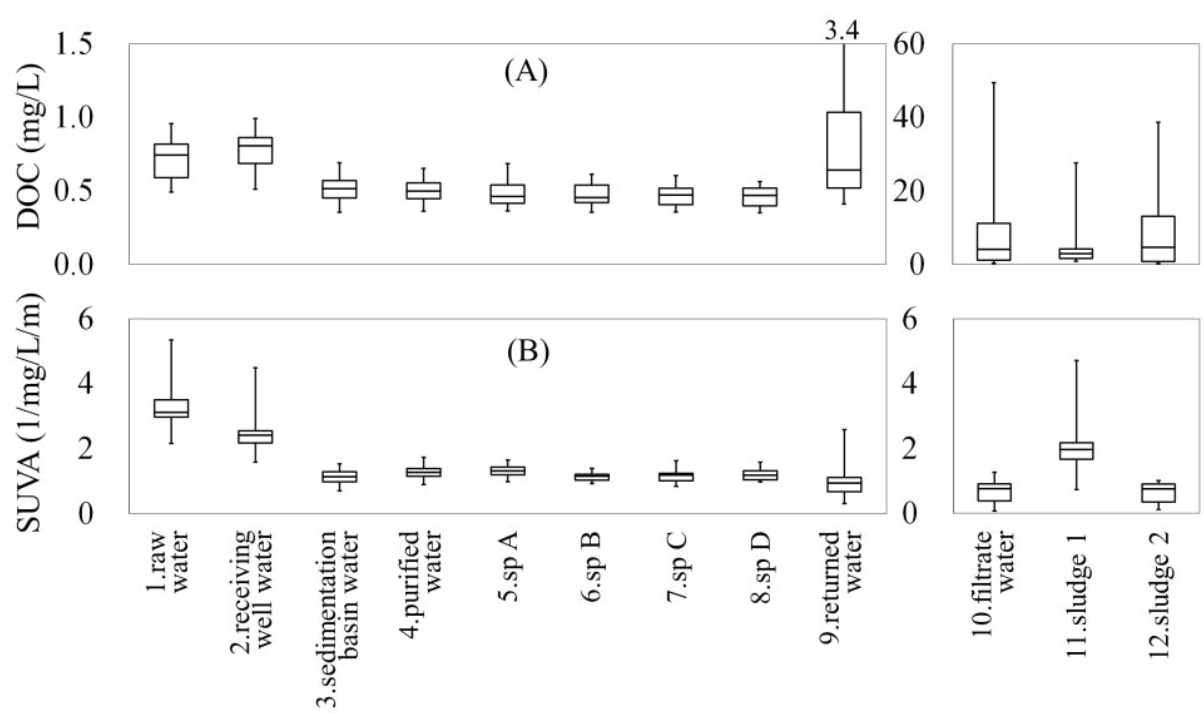

Fig. 2. Boxplots of DOC concentrations (A) and SUVA (B) at the sample sites ( $n=13-22)$.

The results of the EEM measurements exhibit some specific peaks for humic-like and protein-like substances [1] in all water samples from the plant, and the compositions of DOM in the purified water were almost the same as those in raw water (Fig. 3). However, a peak of protein-like substances $(E x / E m=220-230 / 290-295 \mathrm{~nm})[14,15]$ was observed in the purified and returned water, but not in the raw water. 

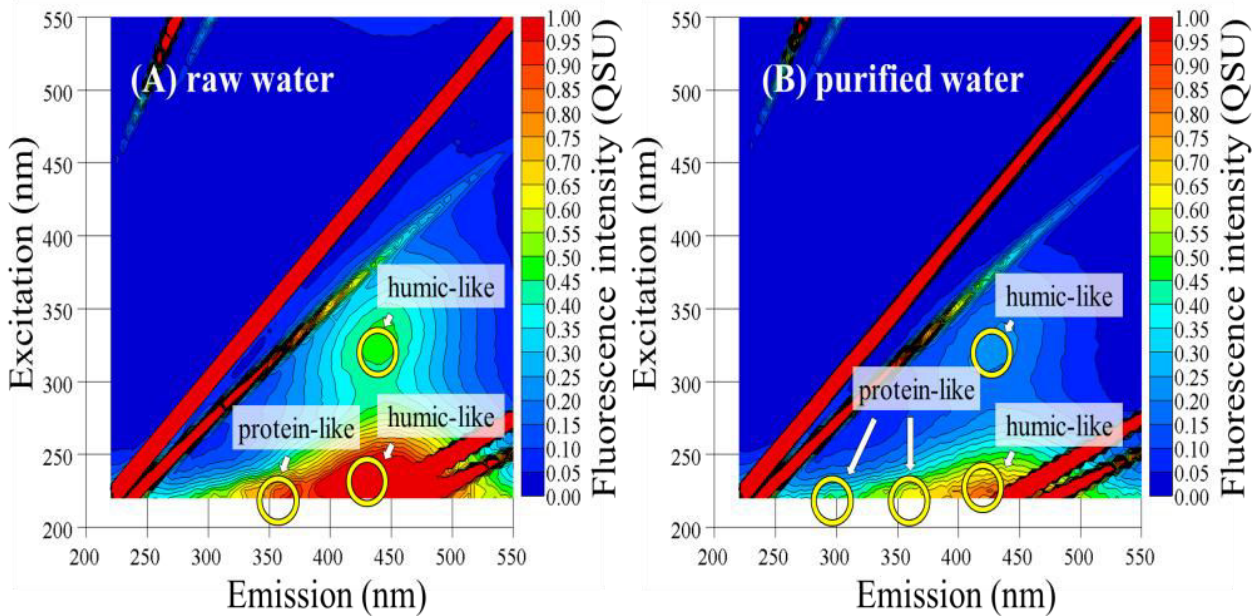

Fig. 3. EEM contour plot of raw water (A) and purified water (B). Samples were collected in August 2016.

The average DON concentrations in the filtrate from the sludge treatment process and raw water were $2.4 \mathrm{mg} / \mathrm{L}$ and $0.07 \mathrm{mg} / \mathrm{L}$, respectively. This suggests that large quantities of DOM were generated in the sludge basin and waste sludge-concentrating tank. The DON concentration of the returned water was approximately $60 \%$ higher than that of raw water. Some specific peaks were observed in the molecular weight distribution of DOM in the samples, as shown in Fig. 4. The intensities of Peak1 (elution time $=24$ minutes) and Peak2 (25 minutes) decreased, whereas those of Peak3 (26 minutes) and Peak5 (32 minutes) did not. The conventional water treatment processes could not easily remove DOM with a smaller molecular weight [3], and a higher coagulant dose is required because the expected removal mechanism is adsorption onto metal hydroxide surfaces [1]. The intensity of Peak5 for filtrate from the sludge treatment process was 26 times higher than that of raw water.

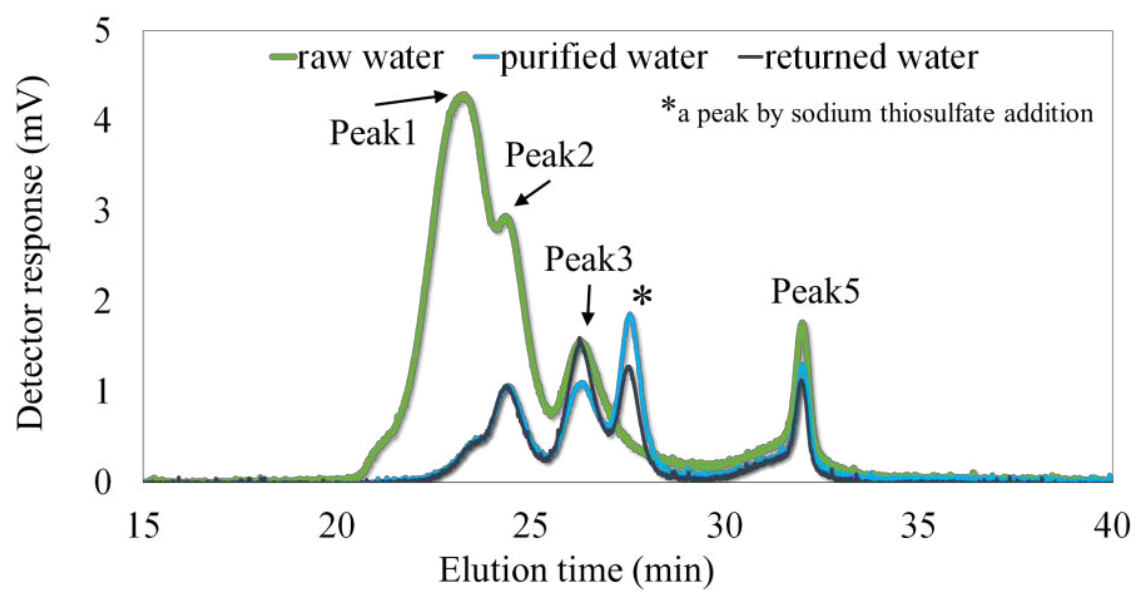

Fig. 4. Molecular weight distribution of the raw, purified, and returned water in August 2016. Peak1, Peak2 = humic substances, Peak3 = building blocks, Peak5 = low-molecular-weight DOM.

Measurement conditions: flow-rate $0.5 \mathrm{~mL} / \mathrm{min}$, detection wavelength $260 \mathrm{~nm}$. 


\subsection{Characteristics of DOM in the distribution pipes}

The average DOC concentrations of the water samples taken at supply points A and D were 0.49 and $0.46 \mathrm{mg} / \mathrm{L}$, respectively. The DOC concentration of the purified water decreased slightly after the water left the plant. The UV260 of the purified water decreased in the distribution pipes. Some specific peaks for humic-like and protein-like substances were present in all water samples from the distribution pipes. The fluorescence intensities of humic-like substances in the purified water decreased soon after the water purification plant, while those of protein-like substances increased as the water traveled from the plant to the pipes. This is expected to be stimulated by residual chlorine, which breaks large molecular compounds down into smaller molecular compounds [9]. The average DON concentrations of the raw water and the water at supply point $\mathrm{D}$ were $0.03 \mathrm{mg} / \mathrm{L}$ and 0.02 $\mathrm{mg} / \mathrm{L}$, respectively. The DON concentration in the purified water decreased slightly in the pipes after the plant. The molecular weight distributions of DOM exhibit some specific peaks in all water samples from the pipes. The intensity of Peak3 from the water at supply point $\mathrm{D}$ did not differ from that of the purified water, whereas the intensity of Peak 5 from the water at supply point $\mathrm{D}$ was approximately $30 \%$ lower than that of the purified water. There were positive relationships between the intensities of Peak5 and residual chlorine decay (Fig. 5), which suggests that DOM is relatively reactive with the residual chlorine in the distribution pipes.

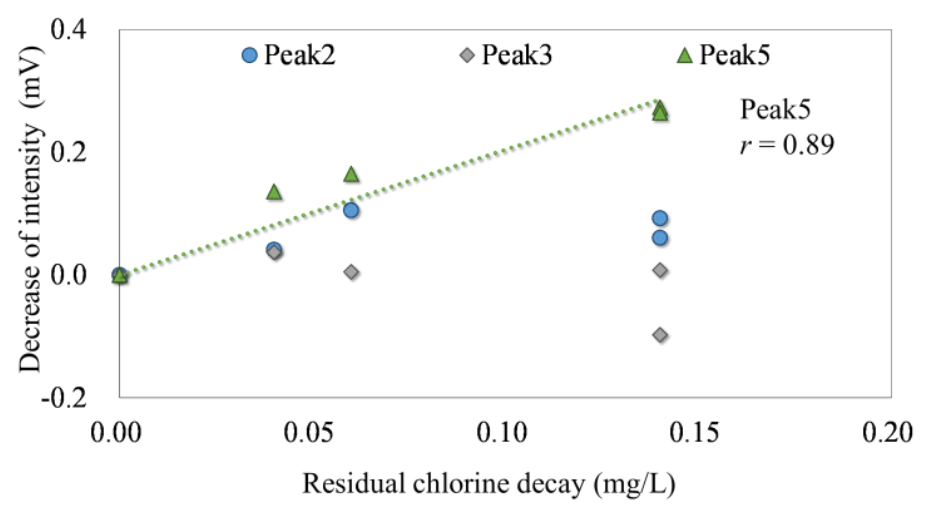

Fig. 5. Relationships between the intensity of water samples from the treatment plant to each supply point and the residual chlorine decay.

\section{Conclusion}

The DOM removed from the raw water undergoing treatment mainly consisted of humiclike substances. The return water could affect the quality of purified water because the DOM characteristics of the return water differed from those of the raw water. The DOM in the purified water could react with the residual chlorine in the distribution pipes.

The authors thank Mr. Daisuke Sougawa and Mr. Takuya Kumazawa for supporting sampling activities and analyses. This work was partially supported by JSPS KAKENHI Grant Number JP17K06616. 


\section{References}

1. M. Sillanpää, Natural Organic Matter in Water, Butterworth-Heinemann (2015)

2. JP. Croué, GV. Korshin, J. Leenheer, and M. Benjamin, AwwaRF (1998)

3. S. So, I. Choi, H. Kim, and S. Maeng, Sci. Total Environ., 592, 584-592 (2017)

4. D. Lee, Y. Cheng, and R. Wong, J. Taiwan Inst. Chem. Eng., 44, 1, 103-110 (2013)

5. J. Haarhoff, M. Kubare, B. Mamba, R. Krause, T. Nkambule, B. Matsebula, and J. Menge, Drink. Water Eng. Sci, 3, 53-61 (2010)

6. C. Jung and H. Son, Korean J. Chem. Eng., 25, 714-720 (2008)

7. I. Kristiana, J. Tan, S. McDonald, CA. Joll, and A. Heitz, Advances in the Physicochemical Characterization of Dissolved Organic Matter: Impact on Natural and Engineered Systems, 209-233 (2014)

8. M. Vera S. Cruz, MR. Boleda, J. Mesa, J. Martín-Alonso, S. Casas, O. Gibert, and JL. Cortina, Sci. Total Environ., 584-585, 1212-1220 (2017)

9. X. Liu, J. Wang, T. Liu, W. Kong, and X. He, PLoS One, 10, 1-11 (2015)

10. W. Li, J. Zhang, F. Wang, L. Qian, Y. Zhou, W. Qi, and J. Chen, Chemosphere, 202, 586-597 (2018)

11. A. Gottfried, AD. Shepard, K. Hardiman, and ME. Walsh, Water Res., 42, 4683-4691 (2008)

12. T. Chen, Y. Xu, Z. Liu, S. Zhu, W. Shi, and F. Cui, J. Environ. Sci., 42, 61-70 (2016)

13. O. Gibert, B. Lefèvre, A. Teuler, X. Bernat, and J. Tobella, J. Water Process Eng., 6, 64-71, (2015)

14. K. Mopper and CA. Schultz, Mar. Chem., 41, 229-238 (1993)

15. LM. Mayer, LL. Schick and TC. Loder III, Mar. Chem., 64, 171-179 (1999)

16. R. Fabris, C. W. K. Chow, M. Drikas, and B. Eikebrokk, Water Res., 42, 4188-4196, (2008) 